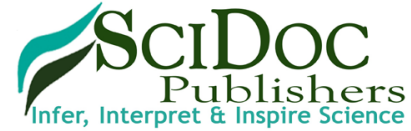

International Journal of Vaccines and Research (IJVR)

ISSN 2572-7427

\title{
Early Clinical Manifestations of Congenital Rubella Syndrome in Oman, 1980-2015
}

Research Article

Al-Awaidy $\mathrm{ST}^{1 *}$, Allison $\mathrm{RD}^{2}$

${ }^{1}$ Communicable Diseases Advisor, Office of H.E. of Health Affairs, Ministry of Health, Muscat, Oman.

${ }^{2}$ Medical Officer, National Institutes of Health, Bethesda, Maryland, USA.

\section{Abstract}

Aim: Congenital rubella syndrome (CRS) is a major cause of severe birth defects worldwide. A national CRS surveillance system was established in Oman to assess the scope of the CRS problem and to detect early manifestations of CRS for planning and early intervention.

Methods: Oman implemented a CRS surveillance system in 2004 that was integrated with measles and rubella case-based surveillance. National surveillance occurs through active and passive reporting from all health institutions. Surveillance guidelines were adopted, including case definitions, an algorithm, standard laboratory methods, system operations, and feedback. A systematic retrospective search was conducted to find and describe CRS cases prior to 2004.

Results: The CRS surveillance system operates in all health facilities and has been integrated with the communicable diseases surveillance system. A total of 104 suspected CRS cases were reported from 1980-2015. Of those, 101 (97\%) were detected through retrospective search (1980-2003) and $3(3 \%)$ through prospective passive surveillance (2004-2015). Of the suspected cases, $61(59 \%)$ were clinically confirmed, 23 (22\%) were laboratory confirmed and $1(1 \%)$ was classified as congenital rubella infection.

Of 85 confirmed cases, 46 (54\%) were located and consented to direct clinical evaluation. In 2015, the median age of this group was 17.9 years (range 8-33 years). Of these patients, 29 (63\%) had ocular, $27(59 \%)$ had auditory, $25(54 \%)$ had neurologic, and $18(39 \%)$ had cardiovascular manifestations of CRS; $11(24 \%)$ had low birth weight $(\leq 2.5 \mathrm{Kg})$ and $3(6 \%)$ had hepatosplenomegaly.

Conclusions: Establishment of a CRS surveillance system that conducts both active and passive surveillance was important to detect and describe CRS. Early clinical manifestations of CRS in Oman included ocular, auditory, neurologic, and cardiovascular abnormalities.

Keywords: Congenital Rubella Syndrome; CRS; Active and Passive CRS Surveillance; Early Manifestations of CRS; Disability; Expanded Program on Immunization; Oman; WHO; Eastern Mediterranean Region.

\section{Introduction}

The World Health Organization (WHO) estimates that more than 110,000 infants are born with congenital rubella syndrome (CRS) each year worldwide [1] CRS can lead to a variety of birth defects, most importantly hearing impairment, congenital heart disease, cataracts, and mental retardation. Many of the CRS manifestations are readily identified during infancy and described as early-onset manifestations [2].

Oman experienced significant rubella epidemics, from 1987 to
1989 and from 1992 to 1996 [Figure 1]. The first dose of rubellacontaining vaccine $(\mathrm{RCV})$ was introduced into the national expanded programme on immunization (EPI) in 1994 as measlesrubella vaccine (MR) at age 15 months. In 1997, MR was replaced by single-dose measles-mumps-rubella vaccine (MMR), and in 2001, a policy of two doses was introduced for MMR at ages 12 and 18 months, both vaccines' coverages were maintained above $>95 \%$ since 1999 . Widespread use of rubella vaccine and a firstdose coverage of more than $95 \%$ led to a dramatic decline in the incidence of rubella and CRS [Figure 1]. Children are screened for their vaccination history at the time of school entry, and nursery/

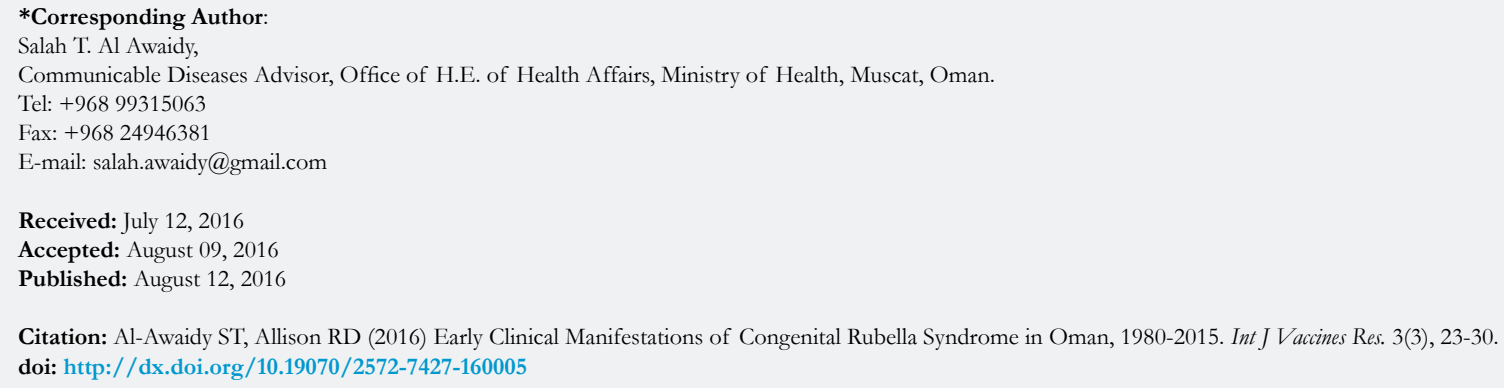

Copyright: Al-Awaidy ST ${ }^{\circ}$ 2016. This is an open-access article distributed under the terms of the Creative Commons Attribution License, which permits unrestricted use, distribu tion and reproduction in any medium, provided the original author and source are credited. 
day-care screening for RCV administration and those lacking evidence of receipt of RCV are referred to the nearest health facility and provided the missing vaccinations. Additionally, since 2001, post-partum rubella vaccination of susceptible mothers with RCV at the time when their infants receive hepatitis $B$ vaccine birth dose and BCG has been a national priority resulting in achievement above 99\% coverage. Newly employed health care workers (HCWs) receive counselling and education about the benefits of vaccination. HCWs are also offered serological testing and free cost vaccination to those who lack evidence of immunity. Further, infection control practices are widely adhered to at delivery, and on paediatric, ophthalmology and neonatology wards [3].

Oman implemented CRS surveillance in 2004, and in 2005, established a policy to eliminate rubella. Rubella elimination was defined as the absence of endemic rubella transmission and no CRS cases associated with importation into Oman for $\geq 12$ months, in the absence of CRS cases associated with endemic transmission and in the presence of a high-quality surveillance system. Oman fully committed to the national elimination goal and strategies and has achieved significant progress towards elimination of rubella and CRS. Since 2000, only 1 case of congenital rubella infection (CRI) was locally transmitted and 2 CRS cases were imported [Figure 1]. This paper describes early manifestations of CRS cases detected by both passive and active surveillance as part of the national CRS surveillance system established in Oman.

\section{Methods}

\section{Congenital Rubella Syndrome surveillance}

In 2004, the CRS surveillance system was implemented in Oman and integrated with measles and rubella case-based surveillance and the national communicable diseases surveillance system. National surveillance reporting is transmitted from all health institutions, private and public, to the governorate communicable diseases surveillance unit. CRS surveillance guidelines were adopted, including case definitions, a surveillance algorithm, standard laboratory methods, system operations, and feedback. The roles of governorate epidemiologists and EPI nurse coordinators were clearly defined including: 1) case and laboratory test result reporting; 2) supervision of the process at all levels within the health services network; and 3) provision of training to HCWs, such as physicians and nurses, on the CRS surveillance guidelines. On-going training of HCWs and periodic reminders about the requirement for timely and zero reporting sent by the Ministry of Health, in particular, have been essential for the successful implementation of the system.

\section{Case ascertainment}

\section{Passive CRS surveillance}

The CRS surveillance system has been included in the reportable diseases group A, the highest priority group, demonstrating its importance to national authorities. This program mandates that every suspected case of CRS must be reported within 24 hours and investigated within 48 hours of identification. All HCWs and health institutions including laboratories, in both the private and public sectors, are required to report CRS cases to their respective governorate communicable diseases surveillance unit.

In this program, the WHO CRS case definitions were utilized, which include: 1) Suspected CRS cases; 2) Clinically confirmed CRS cases; and 3) Laboratory confirmed CRS cases [4].

Suspected CRS: A suspected case is any infant less than one year of age in whom a HCW suspects CRS. HCWs should suspect CRS when an infant presents with heart disease and/or suspicion of deafness and/or one or more of the following eye signs: white pupil (cataract), diminished vision, pendular movement of the eyes (nystagmus), squint, smaller eye ball (microphthalmos), or larger eye ball (congenital glaucoma), or when the infant's mother has a history of suspected or confirmed rubella during pregnancy, even when the infant shows no signs of CRS.

Figure 1. Trend of rubella cases, congenital rubella syndrome (CRS), first and second dose of rubella-containing vaccine (RCV1, RCV2), Oman 1980-2015.

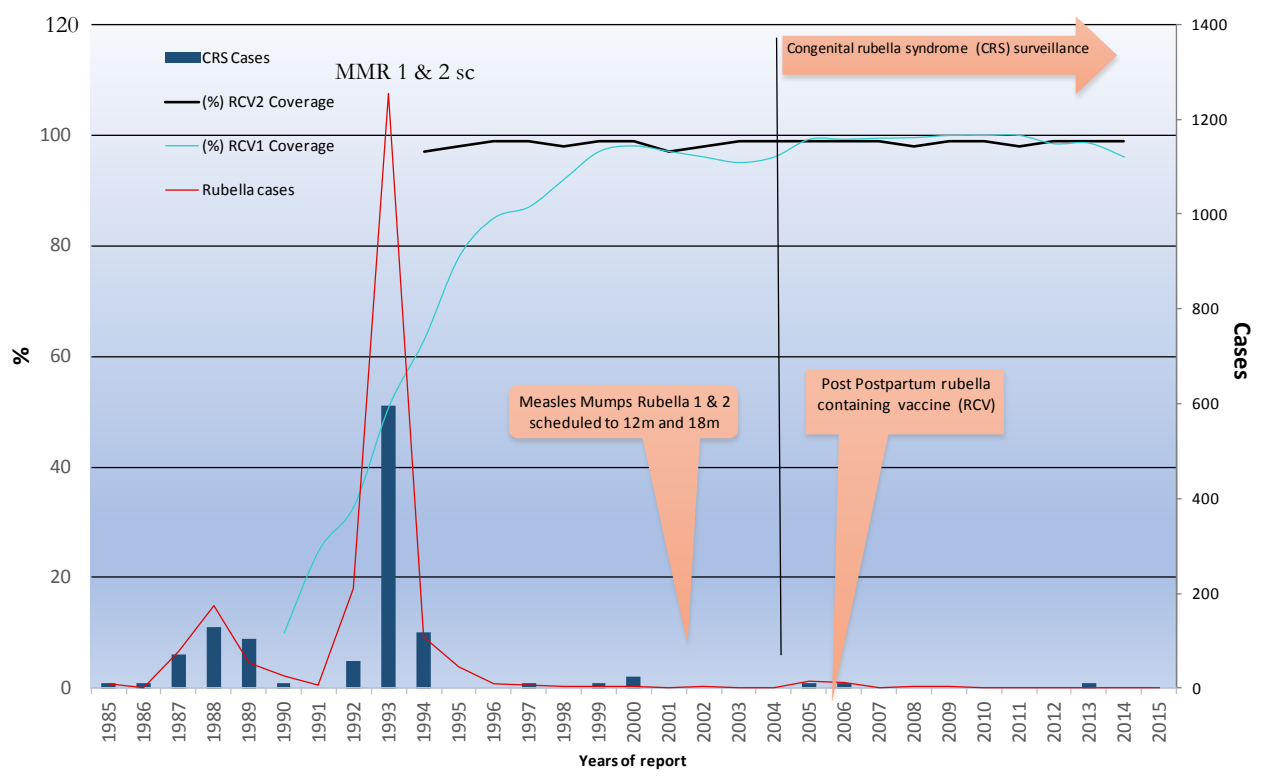


Clinically Confirmed CRS: A clinically confirmed case is an infant in whom a qualified physician diagnoses two or more of the complications from list "A" below or detects one from list $\mathrm{A}$ and one from list " $\mathrm{B}$ " List A includes: cataract(s), congenital glaucoma, congenital heart disease, hearing impairment, or pigmentary retinopathy. List B includes: purpura, splenomegaly, microcephaly, mental retardation, meningo-encephalitis, radiolucent bone disease, or jaundice that begins within 24 hours after birth.

Laboratory Confirmed CRS: A laboratory-confirmed case is an infant with clinically confirmed CRS who has a positive blood test for rubella-specific immunoglobulin M (IgM) obtained as early as possible within the first year of life.

Congenital Rubella Infection (CRI): A case with positive serology, but without manifestations that meet clinically confirmed case criteria [Figure 2].

Discarded Case: A case that does not fulfil the above case definition adopted.

\section{Active CRS Surveillance}

Passive surveillance is supplemented by an active surveillance system that has been conducted using the same infrastructure as for acute flaccid paralysis, neonatal tetanus, measles and rubella surveillance. Governorate epidemiologists visit high priority sites biweekly (two tertiary care sites), medium priority sites monthly (31 secondary governorate $\mathrm{MOH}$ and private hospitals), and low priority sites bimonthly (five sites in private polyclinics, otorhinolaryngology, and paediatric ophthalmology hospitals). The regional epidemiologist directly engages HCWs and staff in paediatrics, labour and delivery, otolaryngology (ENT) and ophthalmology clinics and wards to detect CRS cases. In addition, medical charts and ward log books are reviewed for potential CRSrelated sequelae, including hearing impairment, congenital heart disease, cataracts and blindness. The epidemiologist completes a form on the visit outcome that is signed by both epidemiologist and attending physician and is maintained at both sites. At secondary and tertiary health care facilities thorough hospital medical records of children under one year of age with signs consistent with CRS are scanned using the CRS ICD 10 code to search for any missed CRS cases. In addition, the active surveillance process includes weekly review for suspected CRS cases on the fetal death registry, monthly surveillance reports of congenital anomalies and genetic disorders, inpatient and outpatient statistical data, reports of eye diseases, deafness registry and annual review of medical records of hospitals and handicap centres.

Active surveillance site visits are coupled with refresher training for and sensitization meetings with clinicians and staff nurses. A poster that displays the CRS algorithm for identifying, defining and managing cases is placed in strategic areas in labour and delivery, neonatal and paediatric wards and laboratories to reinforce sensitization. The timelines and completeness of active surveillance visits are monitored monthly by the governorate epidemiologist and the national program manager.

For both passive and active CRS surveillance, the epidemiologists in each governorate determine whether the case meets the surveillance case definition and, if so, the epidemiologist is responsible for gathering necessary epidemiologic data and conducting a follow-up investigation. Surveillance reports are submitted weekly through the acute flaccid paralysis, neonatal tetanus, measles and rubella surveillance structure, with expectation of zero reporting (if no cases are detected, reporting case number as zero).

Feedback on CRS surveillance to health workers is provided

Figure 2. Congenital rubella syndrome (CRS) investigation algorithm, Oman.

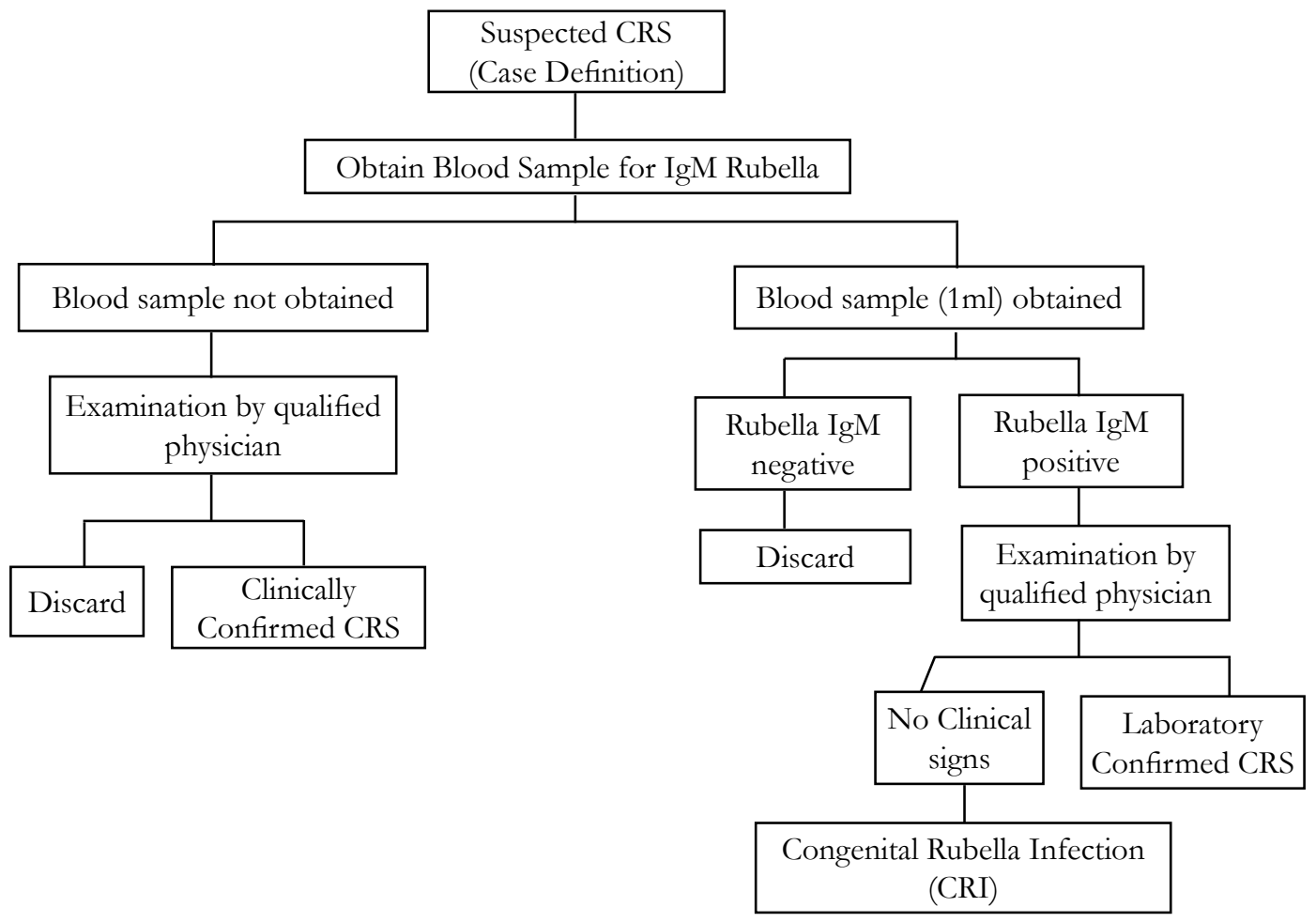


through a monthly national newsletter, and annually through national statistical reports.

\section{Retrospective searches for CRS cases}

To identify CRS cases occurring prior to establishment of CRS surveillance in 2004, a systematic retrospective search for CRS cases was performed. Medical and discharge records were reviewed from 1980-2003 for patients with signs consistent with CRS in healthcare institutions where children with CRS were most likely to have received care, including secondary and tertiary hospitals, specialty clinics and rehabilitation centres. The surveillance case definition for suspected CRS cases was adopted to search other sources including published reports, the fetal death registry, the congenital anomalies and genetic disorders registry, ophthalmological surgery registry, deafness registry and by $\mathrm{HCW}$ interview. All paediatric, neonatology and obstetrics and gynaecology wards at tertiary and secondary hospitals, primary health care clinics with delivery services, ENT and ophthalmology wards, and handicapped and rehabilitation centres, the national deafness prevention programme and civil society organizations were engaged to search for CRS cases. All reports of children less than one year of age at presentation with one or a combination of deafness or hearing impairment, congenital heart disease, cataracts and blindness were investigated.

\section{Case reporting}

The governorate epidemiologist is responsible for completing the surveillance case report form, entering the data in an excel database sheet, and sending the information to the next level each week in accordance with the routine disease notification procedures. Further, the supervision and evaluation of CRS surveillance system operations are conducted in conjunction with that of other communicable diseases surveillance systems, allowing for the identification and strengthening in the deficiencies in the system.

\section{Case investigation including laboratory testing}

All surviving patients with CRS identified retrospectively and prospectively were contacted to schedule detailed clinical assessments using standardized forms. The clinical assessments included a general physical examination and examinations by specialists in ophthalmology, audiology, otorhinolaryngology, paediatrics, cardiology, neurology and endocrinology. The following information was obtained: age, sex, year of birth, history of rash and vaccination during pregnancy or contact with a rubella case, rubella virus testing results and neonatal outcomes [Supplementary Materials, Figure 1]. An ophthalmologist performed a complete eye examination to detect CRS-related ocular manifestations, including a dilated retinal assessment [Supplementary Materials, Figure 2]. Inpatient medical records for CRS patients who had eye surgery were reviewed when available. ENT evaluations included audiologist-performed testing for hearing loss and assessment for speech impairment [Supplementary Materials, Figure 3]. Pure tone audiometric testing was carried out when possible. Hearing impairments were classified as sensorineural, conductive, or mixed. Patients with hearing aids were requested to undergo examination at the ear clinic once every 6 months for the first 15 years and every 5 years thereafter. Speech was assessed for impairments and delays. Each patient with CRS was assessed for a cardiovascular disease history and current cardiac status. Inpatient medical records for CRS patients who have had cardiac surgery were reviewed when available. Each child with CRS was assessed for microcephaly, cerebral palsy, seizure disorder, mental impairment, and any other neurological disease.

Laboratory personnel in each regional public health laboratory are responsible for serum sample collection and shipment to the central public health laboratory (CPHL) for rubella-specific

Figure 3. Congenital rubella syndrome (CRS) surveillance, Oman, 1980-2015.

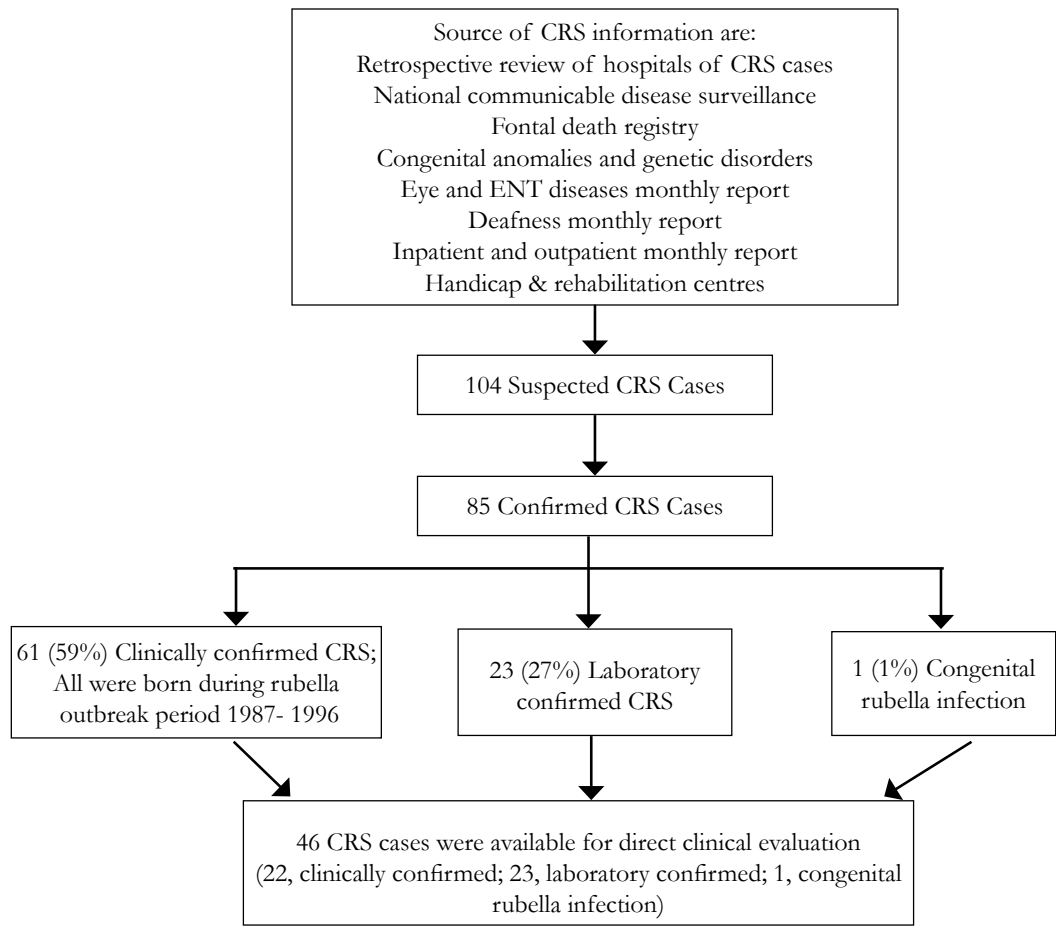


IgM testing by ELISA. The laboratory diagnosis for CRS has been centralized at the national level in the CPHL and is annually validated and accredited with a proficiency test panel performed by the WHO.

\section{CRS surveillance indicators}

CRS surveillance indicators have been monitored nationally and provincially. These include the CRS confirmed case rate per 1000 live births, percentage of suspected cases notified within 24 hours, percentage of suspected cases notified who have had blood samples, percentage of suspected cases notified with a serum sample obtained before six months of age, percentage of notified cases investigated within 48 hours of identification, percentage of notified cases classified by laboratory result, percentage of notified cases with a completed investigation form, percentage of cases with laboratory results within 4 days after receipt in the CPHL and percentage of infants with CRS or CRI who received follow-up within 9 days.

\section{Ethics Approval}

This study was approved by the Oman National Ethical Committee and the National Immunization Technical Advisory Committees.

\section{Results}

\section{CRS surveillance}

CRS surveillance indicators have been monitored since the introduction of CRS surveillance nationally and provincially. During 2000-2015, the annual confirmed mean CRS incidence rate per 1000 live births was zero. The timeliness of reporting and investigation of suspect cases have been on average above $98 \%$ [Table 1]. Timeliness and completeness of the active surveillance visits have been above $95 \%$. In addition, annual validation and

Table 1. Congenital rubella syndrome (CRS) surveillance monitoring indicators, Oman.

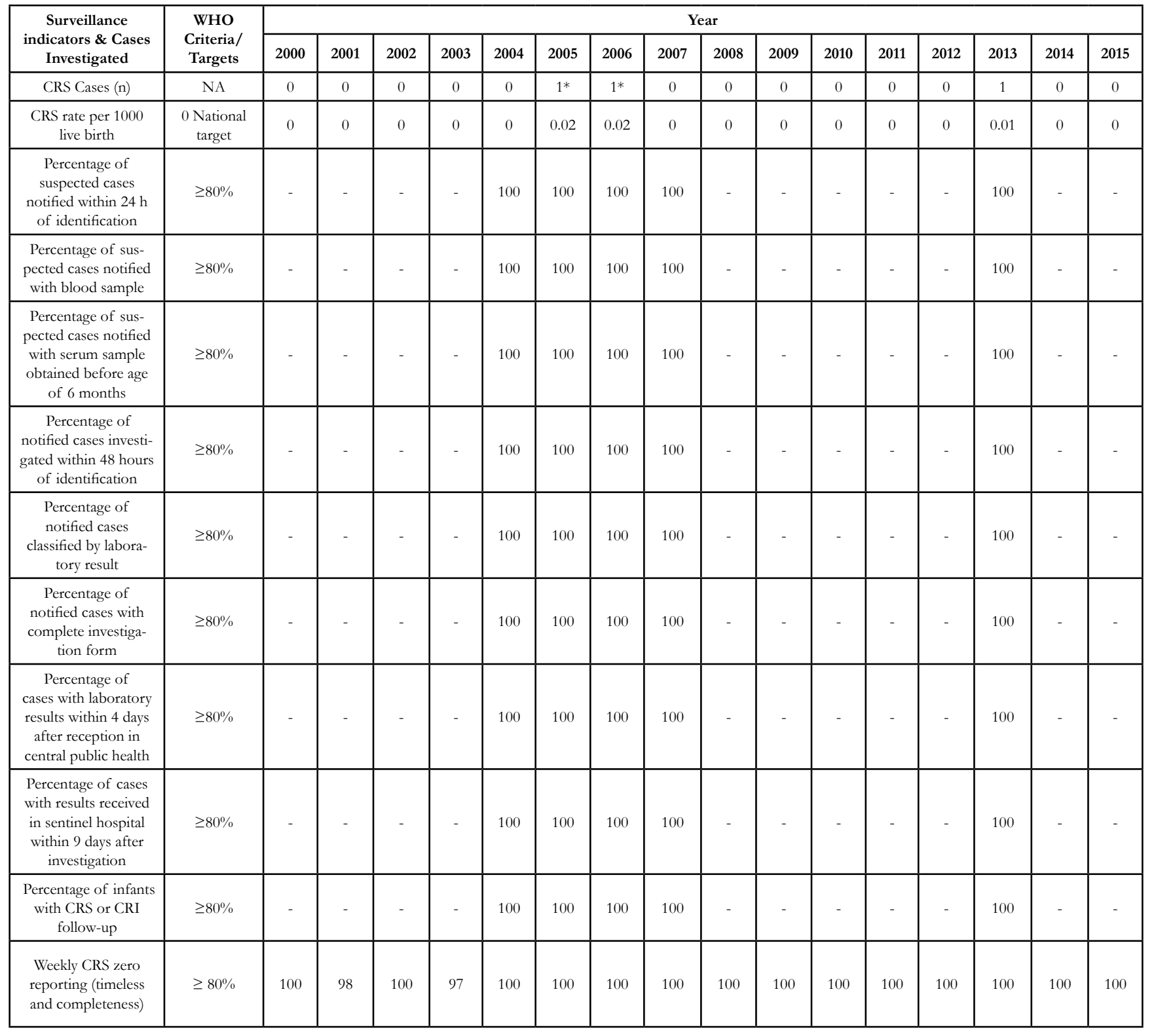

* Imported CRS cases 
accreditation of the CPHL has shown proficiency panel test results consistently above $95 \%$.

A total of 104 suspected CRS cases were reported from 19802015. Of those, 101 (97\%) were detected through retrospective search (1980-1999), 3 (4\%) were detected through prospective passive surveillance ( 2 cases were import-related; 1 was endemic) and none were detected through prospective active surveillance. Eighty-six (83\%) CRS cases were confirmed and 29 (28\%) were discarded [Figure 3]. Of confirmed cases, $61(71 \%)$ were clinically confirmed, $23(27 \%)$ were laboratory-confirmed and $1(1 \%)$ had congenital rubella infection (CRI); $19(22 \%)$ had died and $21(24 \%)$ were lost to follow-up [Table 2]. The 61 clinically confirmed cases were related in place and time to a rubella outbreak from 19871996 in Oman. Of the 19 deceased CRS cases, 7 (37\%) died from cardiovascular causes, 3 (16\%), from meningitis/encephalitis, 2 $(11 \%)$ from pneumonia, $1(5 \%)$, from head injury and $6(31 \%)$ had unknown or unreported causes.

As of 2015, 46 (54\%) CRS-confirmed patients were located and consented for direct clinical evaluation (evaluable). Of those, $28(61 \%)$ were male and $18(39 \%)$ female. In 2015, the median age of this group was 17.9 years (range 8-33 years) [Table 3]. Specialty examinations of the 46 evaluable CRS patients revealed that $29(63 \%)$ had ocular, 27 (59\%) had auditory, $25(54 \%)$ had neurologic, and $18(39 \%)$ had cardiovascular manifestations of CRS; $11(24 \%)$ had low birth weight $(\leq 2.5 \mathrm{Kg})$ and $3(6 \%)$ had hepatosplenomegaly [Table 4a].

Ocular manifestations: $29(63 \%)$ of the evaluable cases had any eye disease, including $14(30 \%)$ with cataract(s) and 5 (11\%) with glaucoma [Table 4b].

Hearing and speech impairment manifestations: 27 $(59 \%)$ patients had hearing loss (81\% bilateral), 21 (46\%) had sensorineural hearing impairment, $1(2 \%)$ had conductive hearing impairment, and $1(2 \%)$ had mixed hearing impairment [Table $4 c]$.
Cardiovascular system manifestations: 18 (39\%) had congenital heart disease, of whom $12(66 \%)$ had patent ductus arteriosus, $4(22 \%)$ had a ventricular septal defect and $1(5 \%)$ had pulmonary stenosis.

Combined CRS manifestations: $12(26 \%)$ had a combination of congenital auditory, ocular and cardiovascular manifestations; $9(20 \%)$ had congenital auditory, ocular and cardiovascular manifestations and microcephaly; and $34(74 \%)$ had congenital auditory, ocular, cardiovascular and neurological manifestations [Table 4a].

\section{Discussion}

Congenital rubella syndrome is a major contributor to the global burden of preventable blindness, deafness, cardiovascular defects and mental retardation [2]. Previous publications from the Oman CRS registry described the implementation of the registry [58], CRS-related ocular manifestations [6] and CRS-related costs [8], but early manifestations of CRS have not been previously described in Oman. In the current study we describe early clinical manifestations after a median of 30 years of follow-up of all evaluable CRS patients from 1980 to 2015 who received comprehensive general and specialty clinical assessments. This is the first description of early CRS manifestations in the Middle East, after prior publications describing CRS in Australia, Canada, Israel and the United Kingdom [9-15]. Early CRS manifestations recorded by the Oman surveillance system included ocular, auditory, neurologic, and cardiovascular manifestations, low birth weight and hepatosplenomegaly. Similar findings were also observed in a number of developed countries [9-15]. Higher prevalence of ocular manifestations $(70 \%)$ observed in these studies compared with our findings (24\%) might be attributed to older mean patient age in these cohorts. While cardiovascular $(31 \%)$ and ocular manifestations $(16 \%)$ were similar to rates in Oman [16].

Table 2. Congenital rubella syndrome (CRS) clinical manifestations identified among evaluable patients, Oman, $1980-2015$.

\begin{tabular}{|c|c|c|c|c|c|}
\hline Detected Period & $\mathbf{1 9 8 0 - 1 9 9 0}$ & $\mathbf{1 9 9 0 - 1 9 9 5}$ & $\mathbf{1 9 9 6 - 2 0 0 0}$ & $\mathbf{2 0 0 1 - 2 0 1 5}$ & Total (\%) \\
\hline CRS patients met case definition & 22 & 57 & 4 & $3^{*}$ & 86 \\
\hline Total deaths & 3 & 16 & 0 & 0 & $19(22)$ \\
\hline Deaths recorded (1980-1993) & 13 & 2 & - & - & $\begin{array}{c}15 / 19 \\
(79 \%)\end{array}$ \\
\hline Lost to follow-up & 14 & 7 & 0 & 0 & $21(24)$ \\
\hline Evaluable CRS patients & 10 & 29 & 4 & $3^{*}$ & $46(54)$ \\
\hline
\end{tabular}

* 2 CRS patients were imported and 1 patient had congenital rubella infection.

Table 3. Age and sex distribution of the evaluable congenital rubella syndrome patients, Oman, 1980-2015.

\begin{tabular}{|c|c|c|c|}
\hline Age Group (Years) & Males & Females & Total \\
\hline $0-12$ & 5 & 1 & 6 \\
\hline $13-19$ & 18 & 11 & 29 \\
\hline $20-29$ & 5 & 6 & 11 \\
\hline Total & $28(61 \%)$ & $18(39 \%)$ & 46 \\
\hline
\end{tabular}


Table 4a. Congenital rubella syndrome (CRS) clinical manifestations identified among evaluable patients, Oman, 1980-2015

\begin{tabular}{|c|c|}
\hline Type of birth defect & $\begin{array}{l}\text { Incidence among evalua- } \\
\text { ble CRS patients, No. (\%) }\end{array}$ \\
\hline Cardiovascular manifestation & $18(39)$ \\
\hline Congenital ocular manifestation & $29(63)$ \\
\hline Congenital auditory manifestation & $27(59)$ \\
\hline Microphthalmia & $7(15)$ \\
\hline Neurological manifestation & $25(54)$ \\
\hline Microcephaly & $24(52)$ \\
\hline Low birth weight $(\leq 2.5 \mathrm{Kg})$ & $11(24)$ \\
\hline Congenital auditory and ocular manifestations & $19(41)$ \\
\hline Congenital auditory, ocular, and cardiovascular manifestations & $12(26)$ \\
\hline $\begin{array}{l}\text { Congenital auditory, ocular, cardiovascular and microcephaly } \\
\text { manifestations }\end{array}$ & $9(20)$ \\
\hline $\begin{array}{c}\text { Congenital auditory, ocular, cardiovascular and neurological } \\
\text { manifestations }\end{array}$ & $34(74)$ \\
\hline Hepatosplenomegaly & $3(6)$ \\
\hline Total evaluable CRS patients & 46 \\
\hline
\end{tabular}

Table 4b. Causes of visual loss among evaluable congenital rubella syndrome patients, Oman, 1980-2015.

\begin{tabular}{|c|c|}
\hline Type of visual lost & No. (\%) \\
\hline Ocular Cataract & $14(48)$ \\
\hline Ocular cataracts one eye & $8(57)$ \\
\hline Ocular cataract both eyes & $6(43)$ \\
\hline $\begin{array}{c}\text { Ocular cataract one } \\
\text { or both eyes }\end{array}$ & $14(100)$ \\
\hline Ocular glaucoma & $5(29)$ \\
\hline Ocular glaucoma one eye & $1(20)$ \\
\hline Ocular glaucoma both eyes & $4(80)$ \\
\hline Retinitis & $16(55)$ \\
\hline Microphthalmos & $6(21)$ \\
\hline Aphakia & $3(10)$ \\
\hline Corneal hydrops & $1(3)$ \\
\hline Hypermetropia & $1(3)$ \\
\hline Keratoconus & $1(3)$ \\
\hline Absorbed lens & $1(3)$ \\
\hline Total ocular defects & 29 \\
\hline
\end{tabular}

The retrospective surveillance and active-case finding greatly facilitated the detection and subsequently description of CRS cases. The establishment of prospective CRS surveillance, adoption of national guidelines, and regular sensitization, including $\mathrm{HCW}$ training, have raised awareness and fostered active participation. High performance on CRS surveillance indicators provide supportive evidence that the Oman CRS surveillance system is of high quality and sensitivity to detect and report endemic and imported cases.

The success of efforts against CRS in Oman is attributable to many factors, including: adoption of CRS elimination goals and strategies; adoption of national guidelines; successful implementation of national CRS surveillance and integration with the existing communicable diseases surveillance system; the availability of dedicated human resources; the capacity of the central laboratory; regular and systematic monitoring; supervision; and evaluation of CRS system performance. Further, establishment of CRS surveillance in Oman facilitated implementation of the CRS elimination strategies aimed at reduction of infant mortality through achievement of high population immunity. At the individual level, the assessments of CRS patients early in life aims to link individuals with CRS and their families to appropriate health professionals and resources for planning and early intervention [7-12].

Of the 19 deaths recorded in the Oman CRS registry, 15 (79\%) were born from 1980 to 1993 and rubella-related heart defects 
Table 4c. Total number of patients with hearing loss among evaluable congenital rubella syndrome patients, Oman, 1980-2015.

\begin{tabular}{|c|c|}
\hline Type of hearing lost & No. (\%) \\
\hline Reporting hearing lost both ears & $22(81)$ \\
\hline Sensorineural hearing impairment & $21(78)$ \\
\hline Conductive hearing impairment & $1(4)$ \\
\hline Mixed hearing impairment & $1(4)$ \\
\hline Unable to test for hearing loss & $3(11)$ \\
\hline Total hearing lost & 27 \\
\hline
\end{tabular}

were reported as the most common contributing cause. This was not unexpected given that correction of major congenital heart defects was not yet available in Oman for children born between 1980-1993. Six CRS-related deaths had no known cause. This is likely attributable to the absence of a standardized central death registry until 2005. Additionally, a common custom is to bury the deceased as soon as possible and this could have occurred without notification since there was no formal requirement to report deaths to health authorities until 1990. This also may have resulted in expiration of some CRS cases soon after birth without entry into the health care system.

One of the study limitations is that the CRS prospective surveillance was not established until 2004 after significant rubella outbreaks in Oman. This limited our ability to complete evaluations on pre-2004 cases and resulted in direct clinical assessments of only $\sim 50 \%$ of patients.

\section{Conclusion}

This paper has highlighted the importance of active and passive CRS surveillance to identify CRS cases in Oman. There was a wide spectrum of observed early clinical manifestations of CRS including ocular, auditory, cardiovascular and neurological abnormalities. Identification of CRS cases provides an opportunity to assess unrecognised manifestations of congenital rubella, understand rubella teratogenesis, support earlier clinical intervention and reduce CRS-related morbidity.

\section{Acknowledgements}

The authors are grateful to the families of the Omani children with CRS for their participation. We are also grateful to H.E. Dr. Ali Bin Moosa, late H.E. Dr. Ali Jaffer Mohammed, late H.E. Dr. Ahmed Al Ghassani, Mr. Hasamudin Mohammed Nwar Alden, Mrs. Halema Al Bulushi, Mrs. Maryam Al Shuabi, Mr. Badder Al Rawahi. Mr. Salim Al Mahrouqi, Mr. Islam Al Bulushi, Mr. Arumugam Raju, Dr. Shyam Bawikar, Dr. Susan Robertson, H.E. Dr. Mohammed Al Hosani, all governorate epidemiologists, NITAG committee members, Dr. Carlos Franco, Dr. Susan Reef and Dr. Gavin Grant for their support and guidance.

\section{References}

[1]. Vynnycky E, Adams, E. J, Cutts F. T, Reef S. E, Navar A. M, et al (2016) Using Seroprevalence and Immunisation Coverage Data to Estimate the Global Burden of Congenital Rubella Syndrome, 1996-2010: A Systematic Review. PloS one, 11(3): e0149160.

[2]. Rubella vaccines: WHO position paper. Wkly Epidemiol Rec. (2011) Jul 15; 86(29): 301-16.

[3]. Meeting of the Strategic Advisory Group of Experts on immunization, November 2013 -- conclusions and recommendations. (2014) Wkly Epidemiol Rec. Jan 3;89(1):1-20.

[4]. Introducing Rubella Vaccine into National Immunization Programmes. A step by step guide (WHO/IVB/15.07). (2015) Sep 3; Accessed on 18 May 2016 at http://apps.who.int/iris/bitstream/10665/184174/1/9789241549370_ eng.pdf.

[5]. Ministry of Health, Sultanate of Oman. Elimination of congenital rubella syndrome: progress towards the goal of elimination by 2005 in Oman. (2004) Community Health \& Disease Surveillance Newsletter 13(1): 1-4.

[6]. Khandekar R, Al-Awaidy S, Ganesh A, Bawikar S (2004) An epidemiological and clinical study of ocular manifestations of congenital rubella syndrome in Omani children. Arch Ophthalmol. 122(4): 541-5.

[7]. Al Awaidy Salah T. et al. Rubella and Congenital Rubella Syndrome Elimination, the Oman Experience. J Vaccine Vaccination. 2015;1(2):9

[8]. Al-Awaidy S, Griffiths U. K, Nwar H. M, Bawikar S, Al-Aisiri M. S, et al (2006) Costs of congenital rubella syndrome (CRS) in Oman: Evidence based on long-term follow-up of 43 children. Vaccine, 24(40): 6437-6445.

[9]. Pritchard C, Schulpher M (2000) Productivity costs: principles and practice in economic evaluation. London.

[10]. Menser MA, Dods L, Harley JD (1967) A twenty-five-year follow-up of congenital rubella. Lancet. Dec 23;2(7530): 1347-50.

[11]. McIntosh ED, Menser MA (1992) A fifty-year follow-up of congenital rubella. Lancet. Aug 15;340(8816): 414-5.

[12]. Forrest JM, Turnbull FM, Sholler GF, Hawker RE, Martin FJ, et al. (2002) Gregg's congenital rubella patients 60 years later. Med J Aust; 177 (11-12): 664-7.

[13]. Munroe S, (1999) A survey of late emerging manifestations of congenital rubella in Canada. Canadian Deaf, Blind and Rubella Association, Brantford, Ontario. http://www.cdbanational.com/PDFs/Congenital\%20Rubella\%20 Study\%20(English).pdf

[14]. Tookey PA, Peckham CS (1999) Surveillance of congenital rubella in Great Britain, 1971-96. BMJ 318: 769-70.

[15]. Desmond MM, Fisher ES, Vorderman AL, Schaffer HG, Andrew LP et al (1978) The longitudinal course of congenital rubella encephalitis in nonretarded children. J Pediatr. Oct; 93(4): 584-91.

[16]. Simons EA, Reef SE, Cooper LZ, Zimmerman L, Thompson KM (2014) Systematic Review of the Manifestations of Congenital Rubella Syndrome in Infants and Characterization of Disability-Adjusted Life Years (DALYs). Risk Anal. 36(7): 1332-56 